\title{
RMS EMITTANCE MEASUREMENTS USING OPTICAL TRANSITION RADIATION INTERFEROMETRY AT THE JEFFERSON LAB FEL*
}

\author{
M. A. Holloway ${ }^{\#}$, R. B. Fiorito, P. G. O'Shea, A. G. Shkvarunets, Institute for Research in \\ Electronics and Applied Physics, University of Maryland, College Park, MD 20742,USA
}

\author{
S. V. Benson, W. Brock, J. L. Coleman, D. Douglas, R. Evans, P. Evtushenko, K. Jordan, D. \\ Sexton, Jefferson Lab, Newport News, VA 23606, USA
}

\begin{abstract}
Optical Transition Radiation Interferometry (OTRI) has proven to be effective tool for measuring rms beam divergence. We present rms emittance measurement results of the $115 \mathrm{MeV}$ energy recovery linac at the Thomas Jefferson National Laboratory's Free Electron Laser using OTRI. OTR data from both near field beam images and far field angular distribution images give evidence of two spatial and angular distributions within the beam. Using the unique features of OTRI we segregate the two distributions of the beam and estimate separate rms emittance values for each component.
\end{abstract}

\section{INTRODUCTION}

Optical transition radiation interferometry has proven to be a capable method for accurate measurements of rms beam divergence in electron beams with energies ranging $10 \mathrm{MeV}$ to $100 \mathrm{MeV}$ [1]. Furthermore, rms divergence measurements taken from a position where the electron beam is focused to its minimum waist can be use to calculate the rms beam emittance [2]. Presented here are results of the application of OTRI diagnostic techniques to the $115 \mathrm{MeV}$ Energy Recovery Linac (ERL) Jefferson Lab Free Electron Laser (FEL). The purpose of this application was to test the unique capabilities of OTRI on a high quality high power electron beam and explore the potential of OTRI to analyze complex beam distributions.

\section{CONCEPT}

The basic concept of OTRI is to place two thin parallel metal foils in the beam line oriented 45 degrees with respect to the beam direction. Forward OTR is produced as the electrons emerge from the first foil and backward OTR is produced as the electrons enter the second foil. The second foil is a reflective surface and the forward and backward OTR interfere and are directed out of the beam line at the angle of specular reflection [1].

The electric field of the electron emerging from the first foil and the photon field of forward OTR destructively interfere until the particles have travelled sufficient distance for the two fields to separate. This distance is referred to as the vacuum coherence length, $\mathrm{L}_{\mathrm{v}}$, and is defined as the distance required for the phase fields of the electron and OTR photon to shift $\pi$ radians. The expression for the vacuum coherence length is

\footnotetext{
*Work supported by the ONR and the JTO

"mholl@umd.edu
}

$$
L_{v}=\frac{\lambda}{\pi\left(\gamma^{-2}+\theta^{2}\right)}
$$

where $\lambda$ is the observation wavelength, $\gamma$ is the Lorentz factor, and $\theta$ is the angle of observation. In order for the backward and forward OTR to interfere, the space between the foils, $d$, is on the order of $L_{v}$.

The spectral-angular distribution of intensity produced by an electron passing through two parallel metal foils can be described by Eq. 2 .

$$
\frac{\partial^{2} I}{\partial \omega \partial \Omega}=\frac{q^{2}}{\pi^{2} c} \frac{\theta^{2}}{\left(\gamma^{-2}+\theta^{2}\right)^{2}} \sin ^{2}\left(\frac{\phi}{2}\right) .
$$

The phase angle is defined as $\phi=d / \mathrm{L}_{\mathrm{v}}$ [2]. The interference term of Eq. 2 is highly sensitive to electron beam parameters. Energy spread, optical bandwidth, and beam divergence all potentially affect the interference fringe visibility. However, if the energy spread is small compared to the divergence, as is the case with the Jefferson Lab FEL [3], the electron beam divergence will dominate the modulation of fringe visibility. The effect of band width is easily mitigated with use of a sufficiently narrow bandwidth band pass filter.

Using a computer code, a Gaussian angular distribution function is convolved with Eq. 2 to produce a theoretical interference pattern. This numerically produced interference pattern can then be fit to a recorded experimental interference profile by adjusting the Gaussian function parameters. The $\sigma$ of the Gaussian is the measured rms beam divergence. A detailed description of the fitting procedure can be found in [1].

\section{EXPERIMENTAL SETUP}

The interferometer was inserted into a 4 way cross along the straight ahead section of the Jefferson Lab FEL ERL after the three accelerating cryogenic modules. The experimental electron beam conditions are found in Table 1.

Table 1: Experimental Beam Conditions

\begin{tabular}{|l|l|}
\hline Beam Energy & $115 \mathrm{MeV}$ \\
\hline Macro Pulse Width & $100 \mu \mathrm{s}$ \\
\hline Micro Pulse rep rate & $2 \mathrm{MHz}$ \\
\hline Charge per bunch & $135 \mathrm{pC}$ \\
\hline Beam Current (Avg) & $150 \mu \mathrm{A}$ \\
\hline
\end{tabular}

T03 Beam Diagnostics and Instrumentation 
OTR light produced by the interferometer is directed out of the cross and into an optical system. The optics are designed to capture images of the far field angular interference pattern and the beam image produced at the surface of the second foil of the interferometer. A schematic of the basic optics design concept is illustrated in figure 1 .

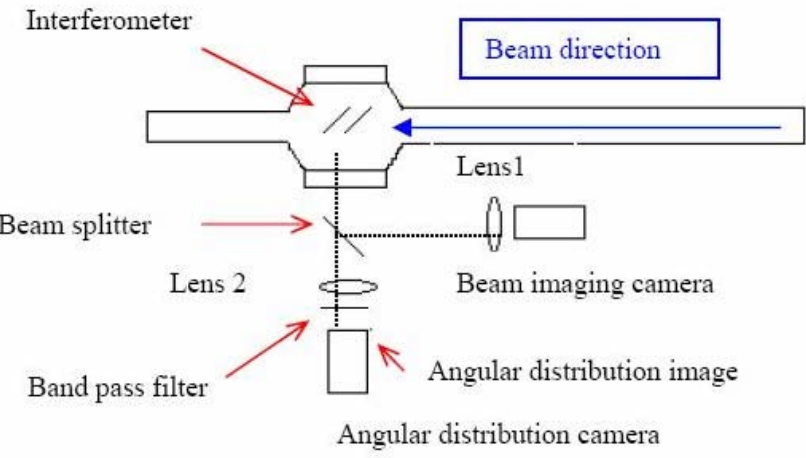

Figure 1: Optical Schematic

Light emerging from the cross strikes a pellicle beam splitter. Ten percent of the light is reflected to a standard RS-170 video CCD camera, which is focused to the reflective surface of the second OTR foil. The remaining light passes through to a highly sensitive 16 bit digital cooled CCD camera that is focused to infinity. A $650 \mathrm{X}$ $10 \mathrm{~nm}$ and a $450 \times 10 \mathrm{~nm}$ band pass filter are placed in the light path before the far field camera.

\section{EXPERIMENTAL RESULTS}

To make emittance measurements, we attempted to focus the electron beam to both a horizontal and vertical waist. At each waist, we acquired an image of the far field interference pattern and an image of the beam profile.

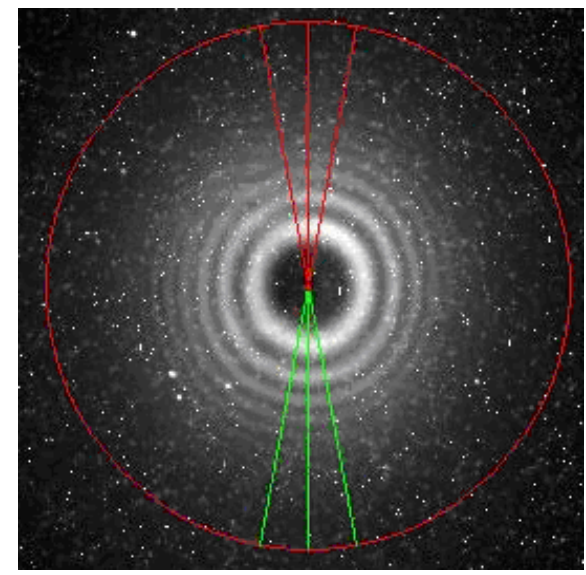

Figure 2: OTRI Interference Pattern at Vertical Waist

\section{Beam Divergence Measurements}

A horizontal and vertical sector scan of the intensity profile is made for each far field image. Within the sector, the pixel values along the same radius from the center of the interference pattern are averaged and an averaged intensity value is assigned for each pixel in the direction of the scan. Averaging the pixel values reduces the effect of noise within the data and provides a smoother curve to perform the fit. Care is taken to minimize the sector angle so that the sector scan is not significantly different than a single line scan through the center of the sector. Figure 2 is the far field interference pattern for the vertical waist condition. The red and green regions in the image define the area of the sector scan.

A proper theoretical fit of the intensity profile could not be achieved using a single Gaussian component for the vertical and horizontal components at each waist condition. In order to get a good fit, two Gaussian components are needed. Figure 3 is a plot of the data fit from the sector scan in Figure 2.

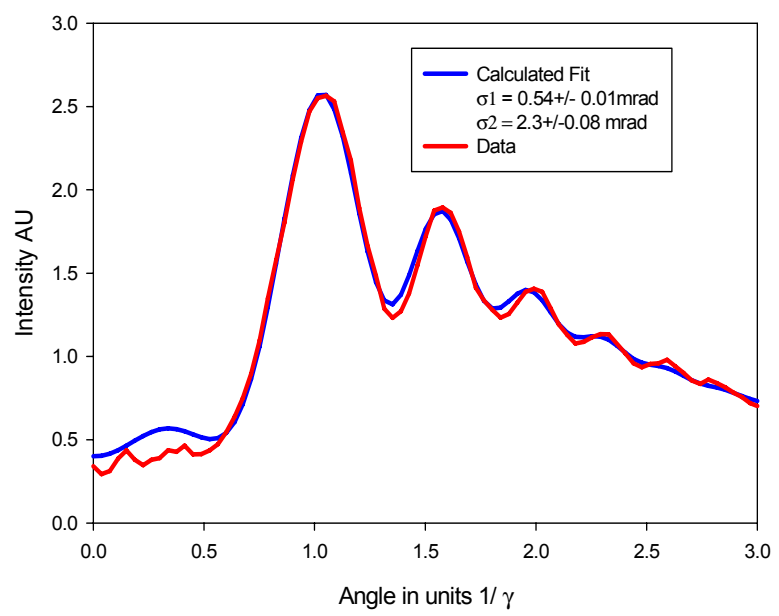

Figure 3: Two Gaussian Fit of the Sector Averaged Scan

The need for a two Gaussian angular distribution function was consistent in the theoretical fits of all the far field data, which suggests that the beam consists of distinct high and low divergent components. The divergence measurements for each beam focus using both filters are presented in Table 2.

Table 2: Divergence Measurements

\begin{tabular}{|l||l|l|l|}
\hline Waist & $\lambda$ & $\theta 1$ (mrad) & $\boldsymbol{\theta 2}$ (mrad) \\
\hline$Y$ & $650 \mathrm{~nm}$ & $0.54+/-.01$ & $2.3+/-0.1$ \\
\hline \hline$Y$ & $450 \mathrm{~nm}$ & $0.55+/-0.01$ & $2.4+/-0.08$ \\
\hline \hline$X$ & $650 \mathrm{~nm}$ & $0.43+/-0.01$ & $1.37+/-0.08$ \\
\hline \hline$X$ & $450 \mathrm{~nm}$ & $0.45+/-0.01$ & $1.28+/-0.07$ \\
\hline
\end{tabular}

\section{Beam Size Measurement}

To obtain a beam size, a Gaussian curve is fit to the intensity profile of the beam waist images. The variance of the fitted curve is used to estimate the rms beam size within an acceptable range of uncertainty.

The beam images show two distinct regions within the spatial distribution. Figure 4 is a beam image of our best focused horizontally focused beam. 


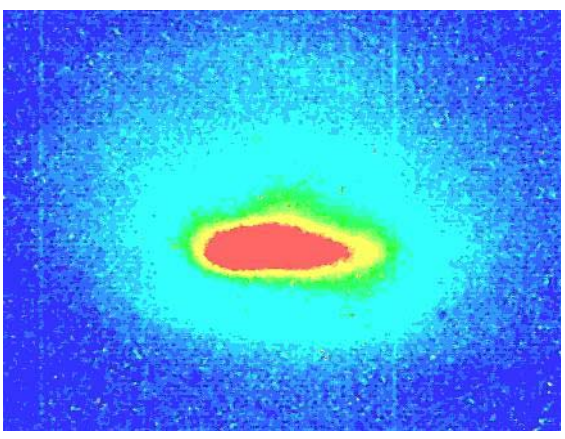

Figure 4: Beam Image

The beam consists of an intense inner peak surround by a lower intensity outer distribution. These beam images also suggest the presence of two distinct distributions within the electron beam. Similar to the fit of the far field data, we used two Gaussian distributions to fit the beam profile.

We use the $\sigma$ of each Gaussian component to estimate the vertical and horizontal beam size of the inner and outer distributions. For each waist, several beam images were taken so that the intensity profiles could be averaged to reduce noise. The results of the measurements are in Table 3.

Table 3: Beam Size Measurements

\begin{tabular}{|l|l||l||l|}
\hline Waist & Wavelength & $\boldsymbol{\sigma 1}(\mu \mathrm{m})$ & $\mathbf{\sigma 2}(\mu \mathrm{m})$ \\
\hline $\mathbf{X}$ & $650 \mathrm{~nm}$ & $134.39+/-1.3$ & $380.09+/-5.61$ \\
\hline $\mathbf{X}$ & $450 \mathrm{~nm}$ & $144.96+/-2.6$ & $508.72+/-16.87$ \\
\hline $\mathbf{Y}$ & $650 \mathrm{~nm}$ & $56.36+/-.59$ & $410.67+/-10.95$ \\
\hline $\mathbf{Y}$ & $450 \mathrm{~nm}$ & $49.43+/-1.01$ & $380.45+/-14.81$ \\
\hline
\end{tabular}

\section{Emittance Measurement}

The normalized rms emittance at a waist condition is given by Eq. 3,

$$
\tilde{\varepsilon}=\gamma \sigma_{r m s} \theta_{r m s}
$$

Where $\sigma$ is the beam size and $\theta$ is the rms divergence in a given dimension [4]. Given that we separated the rms beam size and rms divergence measurements into two components, we estimated the emittance of each component. The results of the calculation are in Table 4.

Table 4: Emittance Calculations

\begin{tabular}{|l||l|l||l|}
\hline Waist & $\lambda$ & $\begin{array}{l}\text { Inner } \boldsymbol{\sigma} \\
\text { (mm-mrad) }\end{array}$ & $\begin{array}{l}\text { Outer } \boldsymbol{\sigma} \\
\text { (mm-mrad) }\end{array}$ \\
\hline $\mathbf{X}$ & $650 \mathrm{~nm}$ & $13+/-.43$ & $117.2+/-7.72$ \\
\hline $\mathbf{X}$ & $450 \mathrm{~nm}$ & $14.7+/-.66$ & $146.5+/-14.02$ \\
\hline $\mathbf{Y}$ & $650 \mathrm{~nm}$ & $6.8+/-.2$ & $212.5+/-14.89$ \\
\hline $\mathbf{Y}$ & $450 \mathrm{~nm}$ & $6.0+/-.23$ & $205.4+/-14.85$ \\
\hline
\end{tabular}

An assumption made in the above calculations is that the low divergence component belongs to the inner beam distribution and that the higher divergence component belongs to the outer beam distribution. There is no way to correspond the position in the spatial distribution with the angular distribution using our current data. The assumption is based on comparison with emittance measurements made by the FEL personnel using conventional methods [3].

In this initial experiment, the waist condition was determined using the naked eye and there was some difficulty in focusing horizontal to minimum. This means that the beam size measurements may be larger than the actual waist size. This will have the effect of increasing the emittance estimate. Also, if there are two distinct beam distributions, it is quite possible that the waist condition for one individual beam distribution may not be the same for the other. This is a possible explanation for the large emittance estimate for the outer beam distribution.

\section{CONCLUSION}

The capability of OTRI to measure complex beam distributions has just begun to be explored. Future experiments will include the use of an optical mask, which will allow light from a particular part of the beam distribution to pass to the far field camera. This will allow us to measure the divergence of small areas of the spatial distribution and allow us to correlate spatial and angular distribution. Optical masks will also be used to perform an all optical method of phase space mapping, which will allow a greater detailed study of complex beam distribution in high power high quality accelerator such as the Jefferson Lab FEL energy recovery linac[5].

\section{REFERENCES}

[1] R.B. Fiorito, et al., "Interference of Diffraction and Transition Radiation and Its Application as a Beam Divergence Diagnostic", Physical Review Special Topics-Accelerators and Beams, vol. 9, 052802, 2006.

[2] R.B. Fiorito and D.W Rule, Ed. Robert E. Shafer , "Optical Transition Radiation Beam Emittance Diagnostics", Beam Instrumentation Workshop, AIP Conf. Proc. no. 319, p. 21 (1994).

[3] Private Communication with D. Douglas (Jefferson Lab Staff Scientist).

[4] M. Reiser, Theory and Design of Charged Particle Beams, New York, NY: John Wiley \& Sons Inc, 1994

[5] R.B. Fiorito, et al., Ed. G.A. Smith and T. Russo "Optical Methods for Mapping the Transverse Phase Space of a Charged Particle Beam", Beam Instrumentation Workshop, AIP Conf. Proc. no. 648. p. 187 (2002). 